\title{
El desafío como estrategia para la promoción de actividad física en universitarios Challenge as a strategy to promote physical activity in university students
}

\author{
Carlos Peñarrubia-Lozano, Lucía Romero-Roso, María Olóriz-Nivela, Manuel Lizalde-Gil
}

Universidad de Zaragoza (España)

\begin{abstract}
Resumen. La práctica regular de actividad física es uno de los elementos que determinan la calidad de vida de las personas. En este sentido, la Organización Mundial de la Salud define como parámetros mínimos para población adulta entre 18 y 64 años la realización de 150 minutos semanales de actividad física aeróbica moderada, 75 minutos de actividad física vigorosa o una combinación equivalente de ambas. En este estudio se ha analizado la influencia que la metodología por desafío presenta en la consecución de dichas recomendaciones. Para ello, han participado 80 estudiantes (13 hombres y 67 mujeres) de los grados universitarios de Magisterio en Educación Primaria (52)

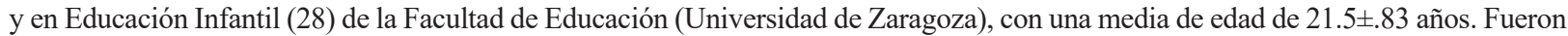
distribuidos aleatoriamente en tres grupos experimentales: grupo uno, con medidas tradicionales de promoción de actividad física; grupo dos, disponiendo de aplicaciones para dispositivos móviles; grupo tres, utilizando pulseras de actividad física. Se utilizó el cuestionario IPAQ para comprobar la cantidad de actividad física realizada. De forma específica se empleó el cuestionario AMPEF para valorar las razones de aumento del ejercicio físico. Los resultados muestran una mejora en el número de estudiantes que cumple con las recomendaciones saludables después de siete semanas. El factor desafío aparece como una razón destacable en los tres grupos experimentales. Los grupos dos y tres destacan la inmediatez de la información facilitada por las herramientas TIC, que permite un mayor control y seguimiento de los retos de práctica planteados.
\end{abstract}

Palabras clave: motivación; salud; OMS; tecnologías de la información y de la comunicación; desafío.

Abstract. Regular practice of physical activity is one of the elements that determine people's quality of life. In this respect, the World Health Organization defines 150 minutes per week of moderate aerobic physical activity, 75 minutes of vigorous physical activity, or an equivalent combination of both, as minimum parameters for the adult population aged between 18 and 64 years old. In this study we analyzed the influence that a challenge methodology has on the achievement of these recommendations. To this end, 80 students ( 13 men and 67 women) from the university degrees in Teaching in Primary School Education (52) and Nursery School Education (28) of the Faculty of Education (University of Zaragoza), with an average age of $21.5 \pm .83$ years old, participated in the study. They were randomly distributed into three experimental groups: group one, with traditional measures to promote physical activity; group two, using applications for mobile devices; group three, using physical activity bracelets. The IPAQ questionnaire was used to collect data on the amount of physical activity performed. Specifically, reasons for increased physical exercise were collected using the AMPEF questionnaire. The results showed an improvement in the number of students fulfilling the healthy recommendations after seven weeks. The challenge factor appears as a remarkable reason in the three experimental groups. Groups two and three highlighted the immediacy of the information provided by the ICT tools, which allows greater control and monitoring of the exercise challenges posed.

Keywords: motivation; health; WHO; information and communication technologies; higher education; challenge.

\section{Introducción}

La práctica de actividad física (PAF) es una herramienta decisiva y eficaz para la mejora de la calidad de vida de las personas, contribuyendo al desarrollo de un estilo de vida saludable (Cecilia, Atucha \& García, 2018; Talaván, 2015; Aranceta, et al., 2016; Rodríguez, Valencia, Gaitán, González \& León, 2017), al presentar diversos beneficios a nivel fisiológico, psicológico y social (De Souza \& Pinheiro, 2017; Padial, Viciana \& Palomares, 2018). A pesar de los beneficios asociados a la PAF, existen numerosos estudios que presentan un alto nivel de sedentarismo e inactividad física (Arboleda, Arango \& Feito, 2016; Jiménez \& Hernández, 2016). No es de extrañar, por tanto, que en la actualidad se considere un tema elemental y prioritario, siendo de especial relevancia en las políticas sociales y de salud (Egeda \& Rodrigo, 2014; Núñez, Zurita, Ramírez, Lozano \& Puertas, 2019), y que organismos internacionales como la Organización Mundial de la Salud (OMS) lleve años proponiendo y actualizando las recomendaciones mundiales sobre actividad física (AF) para la salud de acuerdo a la evidencia científica, incluyendo en ellas las variables de frecuencia, duración, intensidad, el tipo

Fecha recepción: 04-03-20. Fecha de aceptación: 10-05-20 Carlos Peñarrubia-Lozano

carlospl@unizar.es y la cantidad total de actividad física necesaria. De esta manera, este organismo internacional recomienda que los adultos de 18 a 64 años deberían acumular un mínimo de 150 minutos semanales de AF aeróbica moderada, o bien 75 minutos de AF aeróbica vigorosa cada semana, o bien una combinación equivalente de actividades moderadas y vigorosas, aclarando que la AF para este grupo de edades debería consistir en actividades recreativas o de ocio, desplazamientos (por ejemplo, paseos a pie o en bicicleta), actividades ocupacionales (es decir, trabajo), tareas domésticas, juegos, deportes o ejercicios programados en el contexto de las actividades diarias, familiares y comunitarias (OMS, 2010).

En ese amplio rango de la edad adulta, 18 a 64 años, se encuentran los estudiantes universitarios; población que se halla en un período decisivo en la consolidación de un estilo de vida saludable y que también es propensa a perder los hábitos previos de $\mathrm{AF}$, ya que la transición a la vida universitaria puede llegar a ser altamente estresante debido a cambios tan bruscos como cambiar de ciudad y domicilio, aprender a vivir solos, resolver problemas de forma autónoma, conocer a gente nueva, trabajar y convivir con ella, auto administrarse tiempos de estudio y de ocio, etc. (Arias, Solera, Gracia, Silva, Martínez, Cañete \& Sánchez, 2015; Práxedes, Sevil, Moreno, del Villar \& García, 2016; Santos, Alberte, Colmenero, Vázquez, De la Montaña \& Míguez, 2017). Estudios previos determinan que el alumnado universitario pre- 
senta bajos niveles de PAF de acuerdo a las recomendaciones mencionadas anteriormente (Cancela, Ayán, Vila, Gutiérrez \& Gutiérrez-Santiago, 2019; Castañeda-Vázquez, Campos-Mesa \& Castillo-Andrés, 2016; Cocca, Liukkonen, Mayorga \& Viciana, 2014); siendo estos datos más preocupantes en el caso del sexo femenino (Clemente, Nikolaidis, Martins \& Mendes, 2016; Pulgar \& FernándezLuna, 2019). Algunas de las razones aportadas para la no realización de AF de forma regular son: la falta de tiempo; la poca capacidad percibida para la AF; la falta de instalaciones adecuadas para la PAF; la falta de disfrute de la misma; la adherencia a hábitos nocivos como el tabaquismo, alcohol, drogas o mala alimentación; la ausencia de motivación y/o pérdida de compañía de las personas que conforman su red de apoyo; los gastos que puede involucrar practicar AF; el miedo a lesionarse; o barreras ambientales como la inseguridad, la contaminación y la falta de transporte (De Souza \& Pinheiro, 2017; Pérez, Rivera \& Delgado, 2017; Rodríguez, et al., 2017; Sevil, Práxedes, Zaragoza, del Villar \& García, 2017).

Entre los factores que favorecen un estilo de vida saludable y la adherencia a la AF en los estudiantes universitarios destacan las motivaciones de carácter intrínseco como la búsqueda de la propia satisfacción personal, la diversión, sentirse bien con uno mismo gracias a la mejora del estado de salud (Almagro, Sáenz-López, González-Cutre \& Moreno-Murcia, 2011; Deci \& Ryan, 2002; Gómez, Ruiz, García, Granero \& Piéron, 2009; Rico, Arce, Padrón, Peixoto \& Abelairas, 2019), así como la orientación a la tarea y la percepción de un clima motivacional conducente a la misma (Cervelló, Escartí \& Balagué, 1999; Cecchini, González, Carmona \& Contreras, 2004). De esta manera, la motivación surge de la tarea, de la acción, de su dificultad, del problema, del desafío o reto que representa para la persona emprender la acción e intentar finalizarla satisfactoriamente (Orbegoso, 2016; Palmero \& Martínez, 2008).

Por todo ello, en este trabajo se presenta una experiencia piloto llevada a cabo en la Facultad de Educación de la Universidad de Zaragoza, cuyo objetivo principal ha sido impulsar en el alumnado universitario la promoción de la PAF a través de la realización de ejercicio físico $(\mathrm{EF})$, estimulado mediante la metodología de retos y desafíos personales. Los autores estiman que el empleo de herramientas tecnológicas va a favorecer la aparición de resultados positivos al permitir un mayor control de las variables que pueden definir la cantidad y tipo de AF practicada.

\section{Material y método}

\section{Participantes}

El estudio se realizó con metodología cuantitativa, con un diseño con medidas pre y post con el objetivo de comparar tres grupos. En el estudio participó el alumnado matriculado en el turno de mañanas del tercer curso de los grados universitarios de Magisterio en Educación Infantil (MEI) y Magisterio en Educación Primaria (MEP) en la Facultad de Educación (Universidad de Zaragoza). El tipo de muestreo utilizado es no probabilístico y accidental, dado que es de conveniencia, aunque en la configuración de los grupos se respetó la proporcionalidad de los estudiantes matriculados en ambos grados (dos grupos de MEP frente a uno de MEI).
Inicialmente se contó con 105 estudiantes, a los que se dividió en tres grupos de estudio de manera aleatoria: grupo uno, de medidas tradicionales; grupo dos, con uso de aplicaciones para dispositivos móviles (app); y grupo tres, utilizando pulseras de AF. La participación fue voluntaria y todos los estudiantes firmaron un consentimiento firmado. Se excluyeron a todas las personas que no aportaron datos relativos a las mediciones intermedias o la medición final. Con todo ello, la muestra final quedó configurada por 80 estudiantes (13 hombres y 67 mujeres) cuya media de edad fue de $21.5 \pm .83$ años.

\begin{tabular}{|c|c|c|c|c|c|c|c|}
\hline \multirow[b]{2}{*}{ Grado } & \multicolumn{2}{|c|}{ Tradicional } & \multicolumn{2}{|c|}{ App } & \multicolumn{2}{|c|}{ Pulseras de AF } & \multirow[b]{2}{*}{ Total } \\
\hline & Hombre & Mujer & Hombre & Mujer & Hombre & Mujer & \\
\hline MEI & 0 & 8 & 0 & 8 & 0 & 12 & 28 \\
\hline MEP & 4 & 12 & 1 & 12 & 8 & 15 & 52 \\
\hline Total & 4 & 20 & 1 & 20 & 8 & 27 & 80 \\
\hline
\end{tabular}

\section{Instrumentos}

International Physical Activity Questionnaire (IPAQ)

Para medir el nivel de PAF se ha empleado la versión corta del IPAQ(Versión Español-España), IPAQ S7 (Cancela et al., 2019). Este cuestionario permite estimar el tiempo empleado, medido en minutos, para la realización de AF moderada y vigorosa, así como a caminar y a estar sentado. Está compuesto por siete preguntas en las que se abordan cuestiones sobre frecuencia y duración en un día laborable. El sumatorio de los siete días de la semana proporciona una estimación semanal de AF, expresada en equivalentes metabólicos (MET), clasificada en tres niveles: baja $(<600$ Mets* min* sem-1), media (600-2999 Mets* min* sem-1)y alta $\left(>3000\right.$ Mets* min $^{*}$ sem-1).

Autoinforme de Motivos para la Práctica de Ejercicio Físico (AMPEF)

Este instrumento evalúa las razones de práctica de EF. Está conformado por 48 preguntas con respuesta tipo Likert de cero (nada verdadero para mí) a 10 puntos (totalmente verdadero para mí). Las preguntas se agrupan en 11 categorías o factores (F): F1 Peso e imagen corporal, F2 Diversión y bienestar, F3 Prevención y salud positiva, F4 Competición, F5 Afiliación, F6 Fuerza y resistencia muscular, F7 Reconocimiento social, F8 Control del estrés, F9 Agilidad y flexibilidad, F10 Desafío, F11 Urgencias de salud. De acuerdo con Capdevila, Niñerola \& Pintanel (2004), las puntuaciones factoriales son el promedio de las puntuaciones de los ítems que los integran y la puntuación total es el promedio de las puntuaciones de todos los factores (que coincide con el promedio de todos los ítems).

\section{Autoevaluación final}

Se trata de un instrumento ad hoc en el cual los participantes tuvieron que valorar su propio rendimiento en el cumplimiento de los retos semanales planteados por ellos mismos, tanto a nivel individual como colectivo, de acuerdo con el procedimiento establecido y explicado más adelante. Para ello, se pidió expresar una valoración numérica entre uno y 10 , siendo éste el punto de máximo cumplimiento. Se incorporó una pregunta abierta en la que se debían indicar los aspectos que pudieron favorecer o limitar dicho cumplimiento. Finalmente, se dejó un espacio para la inclusión de co- 
mentarios, observaciones y sugerencias.

\section{Aplicaciones para dispositivos móviles}

Los integrantes del grupo experimental dos contaron con dos aplicaciones de similar funcionamiento: 1) Google Fit para el sistema operativo Android y Salud, para IOS; 2) Los acelerómetros y el GPS integrados en los dispositivos móviles facilitan la medición sobre las variables minutos activos, pasos realizados, distancia recorrida y calorías quemadas que permite realizar un seguimiento de la actividad física realizada y comprobar el grado de cumplimiento de los retos semanales.

\section{Pulseras de AF}

Esta herramienta, utilizada por el grupo experimental tres, permite registrar las mismas variables determintantes del tipo y cantidad de AF que en el grupo anterior (minutos activos, pasos diarios, distancia recorrida y calorías quemadas). En este estudio se ha utilizado el modelo MI BAND 3 de Xiaomi. El traspaso de los datos registrados a los dispositivos móviles se ha realizado a través de la aplicación MiFit, compatible con los dos sistemas operativos empleados por los estudiantes (Android, IOS). Las pulseras de AF fueron adquiridas por el Departamento de Expresión Musical, Plástica y Corporal de la Universidad de Zaragoza.

\section{Procedimiento}

Durante el mes de diciembre de 2018 se informó a todos los estudiantes de los grupos seleccionados sobre los objetivos del estudio y la forma de participar en él. Con todos los estudiantes que decidieron participar voluntariamente, se llevó a cabo una primera reunión en el mes de febrero de 2019. En ella se completó el cuestionario IPAQ S7 para determinar la medición del nivel de PAF inicial. Se recordó la metodología de desafío o de planteamiento de retos personales y se distribuyó a los estudiantes en los tres grupos de estudio mencionados anteriormente. Durante la semana siguiente, los estudiantes se comprometieron a hacer un registro de la actividad física diaria para poder plantearse retos de forma individualizada y/o colectiva con el objetivo de mantener o de mejorar su nivel de PAF en las facetas de transporte y tiempo libre, especialmente. Para el grupo experimental uno, de medidas tradicionales, se abrió la posibilidad de participar de forma gratuita en las actividades de Ciclo Indoor, Leg \& Core, Zumba, Body Tono y Fit Boxing realizadas en las instalaciones del Servicio de Actividades Deportivas de la Universidad de Zaragoza. Dichas actividades se desarrollaron dos días por semana, con una duración de 45 minutos por sesión. Además, podían disponer de acceso libre a la sala de musculación, la pista de atletismo y la pista polideportiva. Los grupos experimentales dos y tres debían familiarizarse del mismo modo con las aplicaciones informáticas utilizadas, de cara a poder establecer un reto acorde con la medición de actividad física diaria normal y abordar con garantías el registro de la misma.

La intervención tuvo una duración de siete semanas. Se acordó que al final de cada una de ellas, todos los participantes debían completar una hoja de registro semanal y facilitarla a los investigadores. En ella se debían indicar los minutos activos y el grado de cumplimiento del reto propuesto y, en caso de considerarlo necesario, reajustar o plantear un nuevo compromiso. Además, los grupos experimentales dos y tres debían incorporar los datos complementarios de pasos realizados, distancia recorrida y calorías quemadas, facilitados por las apps y por las pulseras de registro de AF. En la última sesión se completó nuevamente el cuestionario IPAQ $\mathrm{S} 7$ para comprobar el resultado de la intervención. Del mismo modo, y a modo de evaluación, se cumplimentó el cuestionario AMPEF junto con la hoja de autoevaluación.

\section{Análisis de datos}

Para el análisis estadístico se empleó el programa SPSS, versión 25. En primer lugar, se eliminaron los outliers para cada variable. Después, se analizaron la normalidad y la homocedasticidad entre los tres grupos. En aquellas variables que no cumplían algunos de los dos criterios, y estos no se podían corregir, se aplicaron pruebas no paramétricas ( $U$ de de Mann-Whitney). Cuando cumplían los dos criterios, se realizaron las pruebas paramétricas basadas en $t$ de student oANOVAs factoriales (tiempo x condición). Posteriormente se comprobó si había diferencias significativas iniciales entre los tres grupos para cada variable (fiabilidad del 95\%).

Se aplicó un ANOVA simple para comprobar el efecto de cada una de las variables estudiadas (factores del cuestonario AMPEF, autoevaluación, valores del cuestionario IPAQ S7) en función del grupo de estudio. En el caso de las variables del cuestionario IPAQ S7 (minutos de AF moderada; minutos de AF vigorosa; minutos totales de AF; cumplimiento con las recomendaciones de la OMS), se aplicó una ANOVA factorial para conocer si existen diferencias teniendo en cuenta el factor tiempo (pre-post) y el factor condición (pertenencia a grupo tradicional, grupo app o grupo pulseras de AF).

\section{Resultados}

Para garantizar la fiabilidad de los instrumentos se analizó el á de Cronbach entre los diferentes factores señalados en el cuestionario AMPEF (Capdevila, et al., 2004).

Tabla 2

Psicometría de las escalas utilizada

\begin{tabular}{lcc}
\hline Factor de motivación & Facultad Educación & Capdevila et al. (2004) \\
\hline F1 Peso e imagen corporal & $\mathrm{a}=.89$ & $\mathrm{a}=.92$ \\
F2 Diversión y bienestar & $\mathrm{a}=.90$ & $\mathrm{a}=.71$ \\
F3 Prevención y salud positiva & $\mathrm{a}=.81$ & $\mathrm{a}=.84$ \\
F4 Competición & $\mathrm{a}=.88$ & $\mathrm{a}=.90$ \\
F5 Afiliación & $\mathrm{a}=.81$ & $\mathrm{a}=.87$ \\
F6 Fuerza y resistencia muscular & $\mathrm{a}=.82$ & $\mathrm{a}=.83$ \\
F7 Reconocimiento social & $\mathrm{a}=.75$ & $\mathrm{a}=.87$ \\
F8 Control del estrés & $\mathrm{a}=.75$ & $\mathrm{a}=.85$ \\
F9 Agilidad y flexibilidad & $\mathrm{a}=.72$ & $\mathrm{a}=.84$ \\
F10 Desafio & $\mathrm{a}=.80$ & $\mathrm{a}=.85$ \\
F11 Urgencias de salud & $\mathrm{a}=.72$ & $\mathrm{a}=.54$ \\
Basado en Capdevila et al. (2004). & &
\end{tabular}

Basado en Capdevila et al. (2004). física

Tiempo semanal destinado a la práctica de actividad

Para el primer análisis se tuvieron en cuenta las variables contempladas en el cuestionario IPAQ S7, referidas al total de minutos activos y a la cantidad de tiempo semanal dedicada a la práctica de AF moderada y de AF vigorosa. No hay diferencias significativas iniciales entre los tres grupos en la media de minutos activos/semana en la medida pre. En cambio, sí hay diferencias entre los grupos en la medida post, a excepción de la comparativa entre los grupos tradicional y $\operatorname{app}(p=.758)$. En el resto de comparativas, el tamaño del efecto encontrado ha sido muy alto $(p=.000)$. 
Posteriormente se llevó a cabo una ANOVA factorial considerando las condiciones tiempo (medidas pre y post) y grupo (tradicional, app, pulseras de AF). En la comparativa tradicional-app no se encontraron resultados significativos atendiendo a la media de minutos activos por semana, pero sí en el resto de comparativas. Destaca especialmente la comparativa entre los grupos tradicional y pulseras de AF, con diferencias significativas en ambas condiciones, en favor de la medida post $(p=.000)$ y del grupo de pulseras de AF $(p=.000)$.

El análisis respecto a la variable de minutos semanales de AF moderada indica que no hubo diferencias iniciales entre los tres grupos de estudio. Del mismo modo que en la variable anterior de minutos totales de $\mathrm{AF}$ semanal, mediante una ANOVA simple se encontraron diferencias entre los grupos en la medida post $(p=.000)$. En esta ocasión, al aplicar una ANOVA factorial basado en las condiciones de tiempo y grupo de estudio solo se hallaron diferencias significativas en el factor tiempo en las comparativas inter e intragrupos: tradicional-app $(p=.005)$, tradicional-pulseras de $\mathrm{AF}(p=.025)$ $\mathrm{y}$, especialmente, app-pulseras de $\mathrm{AF}(p=.002)$.

En la última de las variables consideradas en este punto, los minutos semanales de AF vigorosa, no se han encontrado diferencias significativas en relación al momento de realización de la medida. Y en el análisis basado en ANOVA factorial, solo han aparecido diferencias significativas en el factor tiempo, con excepción de la comparativa entre el grupo app y el de pulseras de AF. La comparativa entre el grupo tradicional con respecto a los otros dos grupos de estudio presenta los mismos valores de significación y tamaño del efecto $(p=.002)$.

Las variables señaladas sobre el tiempo destinado semanalmente a la realización de AF bien moderada bien vigorosa se tuvieron en cuenta para determinar el número de participantes que cumplen con las recomendaciones de AF saludable sugerida por la OMS. De este modo, en la tabla 3 se puede apreciar que ha habido un incremento en todos los grupos de estudio.

Tabla 3

Cumplimiento de las recomendaciones de la OMS en función del grupo de estudio

\begin{tabular}{llccc}
\hline OMS & Grupo estudio & Pre & Post & $\mathrm{N}$ \\
\hline \multirow{4}{*}{ Cumple OMS } & tradicional & 41.67 & 75.00 & 24 \\
& app & 23.81 & 47.62 & 21 \\
& pulseras de AF & 42.86 & 51.43 & 35 \\
& Total & 37.50 & 57.50 & 80 \\
\hline
\end{tabular}

El siguiente punto del análisis se centra en la comprobación de las razones por las que los participantes dicen practicar EF. Y de una manera más concreta, se quiso analizar la influencia que la metodología por desafío ha tenido para conseguir esta mejora entre los dos momentos en los que se ha llevado a cabo la medición anterior.

\section{Motivos de práctica de ejercicio físico}

Los tres grupos de estudio coinciden en señalar la prevención y salud positiva (factor 3) y la diversión y bienestar (factor 2) como las principales razones por las que realizan EF. El factor desafío, en el que se basa la metodología llevada a cabo en este estudio, aparece como una de las primeras razones para la práctica de EF. Así, es la tercera señalada por los grupos uno y tres, tradicional y pulseras de EF, y la cuarta para el grupo dos, app (tabla 4).

\begin{tabular}{lccc}
$\begin{array}{l}\text { Tabla } 4 \\
\text { Factores de motivación por la realización de AF }\end{array}$ & $\begin{array}{c}\text { Media } \pm \mathrm{DT} \\
\text { tradicional } \\
(\mathrm{n}=24)\end{array}$ & $\begin{array}{c}\text { Media } \pm \mathrm{DT} \\
\text { app } \\
(\mathrm{n}=21)\end{array}$ & $\begin{array}{c}\text { Media } \pm \mathrm{DT} \\
\text { pulseras de AF } \\
(\mathrm{n}=35)\end{array}$ \\
\hline F1 Peso e imagen corporal & $6.53 \pm 2.49$ & $5.67 \pm 2.04$ & $5.86 \pm 1.97$ \\
F2 Diversión y bienestar & $8.00 \pm 1.43$ & $6.37 \pm 2.49$ & $7.36 \pm 1.67$ \\
F3 Prevención y salud positiva & $8.10 \pm 1.45$ & $7.60 \pm 1.49$ & $7.51 \pm 1.46$ \\
F4 Competición & $3.13 \pm 2.96$ & $2.39 \pm 2.20$ & $3.35 \pm 2.57$ \\
F5 Afiliación & $5.67 \pm 2.53$ & $4.62 \pm 1.85$ & $4.49 \pm 2.35$ \\
F6 Fuerza y resistencia muscular & $6.22 \pm 1.49$ & $5.24 \pm 1.24$ & $5.68 \pm 1.37$ \\
F7 Reconocimiento social & $2.21 \pm 2.05$ & $1.85 \pm 1.28$ & $2.88 \pm 1.94$ \\
F8 Control del estrés & $6.44 \pm 2.32$ & $5.87 \pm 2.58$ & $6.20 \pm 2.48$ \\
F9 Agilidad y flexibilidad & $5.13 \pm 2.69$ & $5.98 \pm 1.98$ & $5.77 \pm 2.47$ \\
F10 Desafio & $6.46 \pm 2.19$ & $5.67 \pm 1.91$ & $6.39 \pm 2.46$ \\
F11 Urgencias de salud & $2.08 \pm 2.87$ & $3.25 \pm 2.73$ & $2.48 \pm 2.27$ \\
\hline Basado en Capdevila et al. (2004). & & &
\end{tabular}

Se llevó a cabo un análisis de varianza entre los diferentes factores y las condiciones experimentales, encontrando únicamente diferencias significativas en el factor 2. Diversión y bienestar $(p=.016)$. Posteriormente se realizó un análisis de correlación entre los once factores determinados en el cuestionario AMPEF y las condiciones experimentales. Los factores 7. Reconocimiento social y 11. Urgencias de salud no presentaron correlaciones significativas con ningún otro factor en ninguno de los tres grupos de estudio

A continuación se llevó a cabo un análisis de correlación comparando los grupos entre sí. No se encontraron diferencias significativas en ninguno de los siguientes factores: F3, Prevención y salud positiva; F4, Competición; F5, Afiliación; F7, Reconocimiento social; F8, Control del estrés; F9, Agilidad y flexibilidad. Tampoco en relación a la variable autoevaluación, completada al finalizar el estudio. Atendiendo al factor 10, Desafío, solo presentó diferencias significativas entre los grupos tradicional y app $(p=.015)$ y entre el tradicional y pulseras de AF ( $p=.007)$, no así entre los dos grupos que emplearon herramientas TIC $(p=.655)$. Por otra parte, el factor 1, Peso e imagen corporal, presentó diferencias significativas en la comparativa entre los grupos tradicional y app $(p=.015)$ y tradicional y pulseras de $\mathrm{AF}(p=.007)$. El resto de factores (F2, Diversión y bienestar; F6, Fuerza y resistencia muscular; F11, Urgencias de salud) solo presentó diferencias significativas al comparar los grupos tradicional y app.

Una vez conocido el estudio descriptivo sobre los factores de realización de $\mathrm{EF}$, se ha realizado un análisis de correlación del factor 10, Desafío, respecto al resto de razones de PEF en relación a los tres grupos de estudio. El objetivo pretendido era comprobar si existe alguna asociación de factores que tenga un comportamiento similar en los tres grupos de estudio. De acuerdo con la tabla 5, solo los factores 2, Diversión y bienestar; 6, Fuerza y resistencia muscular; y 9 , Agilidad y flexibilidad presentan esta relación con el factor 10. Desafío en las tres condiciones experimentales. Sin embargo, se puede apreciar que aparecieron diferencias significativas al comparar el desafío con otros factores de forma individualizada para cada grupo de estudio.

Tabla 5

\begin{tabular}{lccc} 
Correlación del factor desafío respecto al resto de variables según el grupo de intervención \\
\hline F10 Desafio & $p$ tradicional & $p$ app & $p$ pulseras de AF \\
\hline F1 Peso e imagen corporal & $.003^{*}$ & .092 & .171 \\
F2 Diversión y bienestar & $.020^{*}$ & $.016^{*}$ & $.005^{*}$ \\
F3 Prevención y salud positiva & $.031^{*}$ & $.003^{*}$ & .663 \\
F4 Competición & $.023^{*}$ & .165 & $.007^{*}$ \\
F5 Afiliación & $.017^{*}$ & .155 & $.046^{*}$ \\
F6 Fuerza y resistencia muscular & $.001^{*}$ & $.000^{*}$ & $.001^{*}$ \\
F7 Reconocimiento social & $.011^{*}$ & .944 & $.001^{*}$ \\
F8 Control del estrés & .655 & $.034^{*}$ & .052 \\
F9 Agilidad y flexibilidad & $.006^{*}$ & $.022^{*}$ & $.000^{*}$ \\
F11 Urgencias de salud & .158 & .254 & .077 \\
\hline
\end{tabular}


Respecto a los resultados obtenidos mediante la autoevaluación, destacan como aspectos facilitadores la gratuidad de los accesos en el grupo uno de medidas tradicionales y la inmediatez del feedback aportado por las herramientas tecnológicas en los grupos dos y tres. Los tres grupos de estudios han señalado como principal fuente de limitación para el cumplimiento de los retos semanales la falta de tiempo. A su vez, los grupos dos y tres han señalado posibles errores de medición o el hecho de tener que incorporar manualmente las estimaciones de AF en situaciones concretas (por ejemplo, en situaciones de partidos oficiales).

\section{Discusión}

Los principales hallazgos de este estudio son: (a) los tres grupos de estudio han incrementado el tiempo semanal de práctica de $\mathrm{AF}$, aunque sigue habiendo un porcentaje elevado de estudiantes que no cumplen con las recomendaciones saludables señaladas por la OMS (2010); b) las motivaciones a la hora de realizar AF han sido principalmente por prevención de salud y por diversión y bienestar; c) el factor desafío aparece como un factor determinante para la realización de AF en los tres grupos de estudio, presentando valores muy superiores a otros estudios (Capdevila et al., 2004). A su vez, se han encontrado correlaciones positivas respecto a los factores diversión y bienestar, fuerza y resistencia muscular y agilidad y flexibilidad.

Los resultados encontrados en este estudio respecto a los estilos de vida saludables de los estudiantes universitarios en relación a la práctica de $\mathrm{AF}$ semanal coinciden con los hallazgos mostrados en trabajos previos (Cocca, et al., 2014; Cocca, Viciana, Mayorga, Ceballos, Liukkonen \& Cocca, 2015; Práxedes, et al., 2016). La etapa universitaria se postula como un período transcendental para la adherencia a la PAF; sin embargo, los estudiantes universitarios son propensos a perder los hábitos previos de $\mathrm{AF}$, ya que la transición a la vida universitaria puede llegar a ser altamente estresante debido a la aparición de desórdenes producidos en el estilo de vida, en ocasiones, siendo un factor primordial en el abandono académico (Santos, et al., 2017). En el presente estudio se ha señalado la falta de tiempo como el principal motivo para no cumplir con los objetivos propuestos semanalmente; datos que coinciden con los encontrados por otros autores (Pérez, et al., 2017; Sevil, et al., 2017), en cuyos estudios también se aprecia un elevado número de jóvenes inactivos y sendentarios. Esta variable ha sido considerada en nuestro estudio mediante el cuestionario IPAQ S7, encontrando que un gran porcentaje de participantes, indiferentemente de la AF realizada, permanecen sentados gran cantidad de horas al día y, además, se desplazan utilizando vehículos motorizados.

A pesar de ello, en la literatura se han encontrado estudios como el de Pedišiæ, Greblo, Phongsavan, Milton \& Bauman (2015) en los que la PAF se asocia significativamente con la satisfacción en la vida, presentando elevados valores de bienestar aquellos universitarios que son físicamente activos. Por tanto, se hace necesaria la implementación y el fomento de hábitos de AF mediante la educación y la promoción de una oferta variada y adaptada a los horarios de la población universitaria, para que los puedan afianzar con el fin de que a lo largo de la vida tengan una mejor salud tanto física como mental (Moreno-Arrebola, Fernández-Revelles, Linares-Manrique \& Espejo-Garcés, 2018).

En relación al cuestionario IPAQ S7, solamente se realizó el análisis del total de minutos activos, de la AF moderada y vigorosa, con el objetivo de que los estudiantes tuviesen una evaluación y una referencia de su nivel de AF inicial. De esta forma, pudieron proponerse los retos individuales y grupales, permitiendo realizar un seguimiento y evaluación del grado de cumplimiento de los mismos. De esta forma, este estudio se ha centrado en la valoración de las razones o motivos por los cuales los estudiantes han decidido incrementar el tiempo de PEF para aumentar ese tiempo de AF total. Conocer los motivos de práctica supone, en este sentido, un factor esencial para crear estrategias motivacionales y retadoras para adherir a los estudiantes de la población universitaria que afirman, en su mayoría, no llevar un estilo de vida activo. De esta forma, saber las motivaciones individuales que llevan a los estudiantes a la realización de AF constituye la base para la implementación de políticas eficaces en la promoción de PAF (Leyton, García, Fuentes \& Jiménez, 2018). La motivación se relaciona en el proceso de adaptación en la PAF, donde se incluyen percepciones de competencia, autoestima, rendimiento, formas intrínsecas de regulación motivacional y estados afectivos, entre otros (Harwood, Keegan, Smith \& Raine, 2015).

Fijando el foco en la motivación, en nuestro estudio los motivos principales señalados por los estudiantes para la realización de AF han sido principalmente la prevención de salud y la diversión y el bienestar. Los datos coinciden con los hallazgos de Rico (2019), en donde la población universitaria consideró que los motivos que más inciden en la PAF son: hacer ejercicio físico, mantener y/o mejorar la salud, satisfacción personal, diversión y pasar el tiempo, el gusto por realizar AF y, finalmente, escapar de la rutina. Ya en el estudio de Capdevila et al. (2004), los sujetos activos mostraron una motivación intrínseca mayor que los sedentarios, puntuando más alto los motivos de diversión o bienestar, de control del estrés o de mejora de la condición física. Además, el factor desafío obtuvo una puntuación media (4.27) por debajo de la encontrada en nuestro estudio (6.46 en el grupo tradicional; 5.67 en el grupo app; 6.39 en el grupo pulseras de AF). De esta forma, se puede afirmar que la metodología por desafío con la que se ha abordado este estudio ha incidido positivamente en la mejora de los datos de PAF. Los retos semanales parecen favorecer la adherencia a la AF, permitiendo mejorar la calidad de vida de los participantes. Como aspectos facilitadores, la gratuidad de acceso a las instalaciones ofertada para el grupo de medidas tradicionales aparece como el principal motivo para explicar el aumento en el tiempo destinado a la PAF, coincidiendo con otros estudios (Tauler, et al., 2017). Por otra parte, experiencias previas sugieren que el uso de dispositivos tecnológicos como apps y pulseras de medición de AF contribuyen a la mejora del tiempo destinado a la práctica de AF semanal o bien al mantenimiento de estilos activos si los estudiantes ya los han adquirido (Aroni, et al., 2017; Bice, Ball, Adkins \& Ramsey, 2016; Gabbiadini \& Greitemeyer, 2018; Maxwell, et al., 2018; Pulido, Sánchez, Sánchez, González \& García, 2016; Rosales, Fernández, Comunello, Mulargia \& Ferran, 2017). No obs- 
tante, no se encontraron estudios previos sobre la fiabilidad de las aplicaciones para dispositivos móviles, tampoco para las pulseras de actividad física. La disposición de un feedback personalizado e inmediato es el principal argumento señalado por los participantes en los grupos experimentales dos y tres de nuestro estudio, del mismo modo que los datos encontrados en trabajos previos (Tong, Coiera \& Laranjo, 2018).

Con todo ello, se puede concluir que todas las intervenciones desarrolladas a través de la metodología del reto o desafío han supuesto un incremento del volumen en la PAF de los estudiantes, manifestada especialmente en un aumento del tiempo destinado a la realización de EF, adecuándose de esta manera a las recomendaciones de la OMS.

Este trabajo presenta algunas limitaciones atendiendo a la muestra participante, ya que no es representativa del total del alumnado objeto de estudio. Los resultados atienden únicamente a los tres grupos de tercer curso matriculados en la franja de mañanas (con un margen de error del 10\%), cuando en esta Facultad se cuenta con otros tantos grupos que cursan sus estudios en la franja de tardes. Para nuevos estudios podría ser interesante ampliar el número de participantes, contando con una muestra representativa de todos los grupos y cursos de los dos grados universitarios. Del mismo modo, se deberá tener en cuenta la proporcionalidad de la muestra en relación tanto al sexo como al grado universitario al que pertenezcan los estudiantes.

\section{Conclusiones}

Todos los grupos de estudio presentan una mejora en el número de estudiantes que cumple con las recomendaciones saludables mínimas definidas por la OMS para población adulta. Este hecho se ha alcanzado gracias a un mayor tiempo semanal destinado a la PAF, especialmente en relación a la AF moderada. A pesar de estos datos positivos, el $42.5 \%$ de los estudiantes sigue sin alcanzar los parámetros mínimos considerados saludables. Respecto a las razones por las que los participantes afirman realizar EF para aumentar el tiempo de PAF, destacan como principales argumentos la diversión y bienestar y la prevención y salud positiva. El reto o desafío personal aparece como una razón importante en los tres grupos experimentales, encontrándose a su vez una correlación positiva entre el factor desafío y los factores diversión y bienestar, mejora de la fuerza y de la resistencia muscular y ganancia de agilidad y flexibilidad. La inmediatez del feedback otorgado por las herramientas tecnológicas favorece el control y seguimiento de los retos personales.

\section{Agradecimientos}

Los autores del estudio quieren agradecer el apoyo institucional recibido por la Facultad de Educación y el Vicerrectorado de Cultura y Proyección Social y dentro del mismo, al Servicio de Actividades Deportivas por la colaboración desinteresada en el ofrecimiento de sus instalaciones $\mathrm{y}$ actividades.

\section{Referencias}

Almagro, B. J., Sáenz-López, P., González-Cutre, D., \& MorenoMurcia, J. A. (2011). Clima motivacional percibido, necesida- des psicológicas y motivación intrínseca como predictores del compromiso deportivo en adolescentes. Revista Internacional de Ciencias del Deporte, 25, 250-265.

Aranceta, J., Arija, V., Maíz, E., Martínez, E., Ortega, R. M., Pérez, C., ... Serra, L. (2016). Guías alimentarias para la población española (SENC, diciembre 2016); la nueva pirámide de la alimentación saludable. Nutrición Hospitalaria, 33, 1-48. https:/ /doi.org/10.20960/nh.827.

Arboleda, V. H., Arango, E. F., \& Feito, Y. (2016). Actividad física y percepciones de beneficios y barreras en una universidad colombiana. Retos. Nuevas tendencias en Educación Física, Deportes y Recreación, 30, 15-19.

Arias, N. M., Solera, M., Gracia, L., Silva, P., Martínez, V., Cañete, J., \& Sánchez, M. (2015). Levels and Patterns of Objectively Assessed Physical Activity and Compliance with Different Public Health Guidelines in University Students. PLoS ONE, 10(11), 1-15. https://doi.org/10.1371/journal.pone.0141977.

Aroni, A., Castillo, E., Sousa, C., Machado, A., Filho, E., \& Tenenbaum, G. (2017). Smartphone Applications Used for Initiating and Maintaining. Physical Activity: An Exploratory Analysis. Journal of Sport Psychology, 27(1), 89-95.

Bice, M. R., Ball, J., Adkins, M. M., \& Ramsey, A. (2016). Health Technology Ownership and Use: Implications for Adult Physical Activity. Journal of Sport and Health Research, 8(1), 13-22.

Cancela, J. M., Ayán, C., Vila, H., Gutiérrez, J. M., \& GutiérrezSantiago (2019). Validez de constructo del Cuestionario Internacional de Actividad Física en universitarios españoles. RIDEP, 52(3), 5-14. https://doi.org/10.21865/RIDEP52.3.01.

Capdevila, L., Niñerola, J., \& Pintanel, M. (2004). Motivación y actividad física: el autoinforme de motivos para la práctica de ejercicio físico (AMPEF). Revista de Psicología del Deporte, 13(1), 55-74.

Castañeda-Vázquez, C., Campos-Mesa, M. C., \& Del CastilloAndrés, O. (2016). Actividad física y percepción de salud de los estudiantes universitarios. Rev. Fac. Med., 64, 277-84. http:/ /dx.doi.org/10.15446/revfacmed.v64n2.53068.

Cecchini, J. A., González, C., Carmona, A. M., \& Contreras, O. (2004). Relaciones entre clima motivacional, la orientación de meta, la motivación intrínseca, la autoconfianza, la ansiedad y el estado de ánimo en jóvenes deportistas. Psicothema, 16, 104-109.

Cecilia, M. J., Atucha, N. M., \& García, J. (2018). Estilos de salud y hábitos saludables en estudiantes del Grado en Farmacia. Educación Médica, 19(S3), 294-305. https://doi.org/10.1016/ j.edumed.2017.07.008.

Cervelló, E., Escartí, A., \& Balagué, G. (1999). Relaciones entre la orientación de meta disposicional y la satisfacción con los resultados deportivos, las creencias sobre las causas de éxito en el deporte y la diversión con la práctica deportiva. Revista de Psicología del Deporte, 8, 7-21.

Clemente, F. M., Nikolaidis, P. T., Martins, F. M., \& Mendes, R. S. (2016) Physical Activity Patterns in University Students: Do They Follow the Public Health Guidelines? PLoS ONE, 11(3), 1-11. https://doi.org/10.1371/ journal.pone. 0152516 .

Cocca, A., Liukkonen, J., Mayorga, D., \& Viciana, J. (2014). Healthrelated Physical Activity levels in Spanish youth and young adults. Perceptual and Motor Skills, 118(1), 247-260. https:// doi.org/10.2466/10.06.PMS.118k16w1

Cocca, A., Viciana, J., Mayorga, D., Ceballos, O., Liukkonen, J., \& Cocca, M. (2015). Adolescents' and young adults' physical activity related to built environment. Educación Física y Ciencia, 17(1), 1-11.

Craig, C. L., Marshall, A. L., Sjöström, M., Bauman, A. E., Booth, M. L., Ainsworth, B. E., ..., Oja, P. (2003). International 
Physical Activity Questionnaire: 12-Country Reliability and Validity. Med Sci Sports Exerc, 35(8), 1381-1395. DOI: http:/ /dx.doi.org/10.1249/01.MSS 0000078924.61453.FB

Deci, E. L., \& Ryan, R. M. (2000). Self-Determination Theory and the Facilitation of Intrinsic Motivation, Social Development, and Well-Being. American Psychologist, 55(1), 68-78. doi: 10.1037110003-066X.55.1.68.

De Souza, C., \& Pinheiro, A. (2017). Mudanças na atividade física de universitários: associação com informações sobre saúde e acesso a locais para prática. Revista Salud Pública, 5(19), $617-$ 623. doi:http://dx.doi.org/10.15446/rsap.v19n5.46072.

Egeda, J. M., \& Rodrigo, M. (2014). Adherencia a la Dieta Mediterránea en futuras maestras. Nutrición Hospitalaria, 30(2), 343350. doi: http://dx.doi.org/10.3305/nh.2014.30.2.7585.

Gabbiadini, A., \& Greitemeyer, T. (2018). Fitness mobile apps positively affect attitudes, perceived behavioural control and physical activities. Journal of Sports Medicine and Physical Fitness, 4, 1-21. doi:10.23736/S0022-4707.18.08260-9.

Gómez, M., Ruiz, F., García, M. E., Granero, A, \& Pieron, M. (2009). Motivaciones aludidas por los jóvenes universitarios que practican actividades físico-deportivas. Rev Lat Am Psicol, 41(3), 519-32.

Harwood, C. G., Keegan, R. J., Smith, J. M. J., \& Raine, A. S. (2015). A systematic review of the intrapersonal correlates of motivational climate perceptions in sport and physical activity. Psychology of Sport and Exercise, 18, 9-25. doi: http:// dx.doi.org/10.1016/j.psychsport.2014.11.005.

Jiménez, S., \& Hernández, J. (2016). Actividad Física y otros hábitos de vida de estudiantes de medicina de la Universidad de Costa Rica. PENSAR EN MOVIMIENTO: Revista de Ciencias del Ejercicio y la Salud, 14(1), 1-14. doi: 10.15517/ PENSARMOV.V14I1.19294.

Leyton, M., García, J., Fuentes, J. P., \& Jiménez, R. (2018). Análisis de variables motivacionales y de estilos de vida saludables en practicantes de ejercicio físico en centros deportivos en función del género. Retos. Nuevas Tendencias en Educación Física, Deportes y Recreación, 34, 166-171.

Maxwell, C., Cohen, P., Platell, C., Tan, P., Levitt, M., Salama, P., ... Hardcastle, S. J. (2018). Wearable Activity Technology And Action-Planning (WATAAP) to promote physical activity in cancer survivors: Randomised controlled trial protocol. International Journal of Clinical and Health Psychology, 18, 124- 132. doi: 10.1016/j.ijchp.2018.03.003.

Moreno-Arrebola, R., Fernández-Revelles, A. B., LinaresManrique, M., \& Espejo-Garcés, T. (2018). Revisión sistemática sobre hábitos de actividad física en estudiantes universitarios. Sportis. Revista Técnico-Científica del Deporte Escolar, Educación Física y Psicomotricidad, 4(1), 162- 183. https:// doi.org/10.17979/sportis.2018.4.1.2062

Núñez, J., Zurita, F., Ramírez, I., Lozano, M., y Puertas, P. (2019). Análisis de la relación entre los hábitos físico-saludables y la dieta con la obesidad en escolares de tercer ciclo de Primaria de la Provincia de Granada. Retos. Nuevas Tendencias en Educación Física, Deportes y Recreación, 35, 31-35.

Orbegoso, A. (2016). La motivación intrínseca según Ryan y Deci y algunas recomendaciones para maestros. Educare, Revista Científica de Educação, 2(1), 75-93. http://dx.doi.org/10.19141/ 2447-5432/lumen.v2.n1.p.75-93.

Organización Mundial de la Salud, OMS. (2010). Recomendaciones mundiales sobre actividad física para la salud. Suiza: Autor.

Padial, R., Viciana, V., \& Palomares, J. (2018). Adherencia a la dieta mediterránea, la actividad física y su relación con el IMC, en estudiantes universitarios del grado de primaria, mención de educación física, de Granada. ESHPA-Education, Sport, Health and Physical Activity, 1(2), 30-49. doi: http://hdl.handle.net/
$10481 / 49836$.

Palmero, F.; \& Martínez, F. (2008). Motivación y emoción. Madrid: McGraw-Hill.

Pedišiæ, Z., Greblo, Z., Phongsavan, P., Milton, K., \& Bauman, A. E. (2015). Are total, intensity-and domain-specific physical activity levels associated with life satisfaction among university students? Plos One, 10(2), 1-21. https://doi.org/10.1371/ journal.pone. 0118137 .

Pérez, I. J., Rivera, E., \& Delgado, M. (2017). Mejora de hábitos de vida saludables en alumnos universitarios mediante una propuesta de gamificación. Nutrición Hospitalaria, 34(4), 942951. https://dx.doi.org/10.20960/nh.669.

Práxedes, A., Sevil, J., Moreno, A., del Villar, F., \& García, L. (2016). Niveles de actividad física y motivación en estudiantes universitarios. Diferencias en función del perfil académico vinculado a la práctica físico-deportiva. Journal of Sport and Health Research, 8(3), 191-204.

Pulgar, S., \& Fernández-Luna, A. (2019). Práctica de actividad física, consumo de tabaco y alcohol y sus efectos en la salud respiratoria de los jóvenes universitarios. Retos. Nuevas Tendencias en Educación Física, Deportes y Recreación, 35, 130135.

Pulido, J. J., Sánchez, D., Sánchez, P. A., Gónzalez, I., \& García, T. (2016). Proyecto MÓVIL-ÍZATE: Fomento de la actividad física en escolares mediante las Apps móviles. Retos. Nuevas Tendencias en Educación Física, Deportes y Recreación, 30, 38.

Rico, J., Arce. C., Padrón, A., Peixoto, L., \& Abelairas, C. (2019). Motivaciones y hábitos de actividad física en alumnos universitarios. Retos. Nuevas Tendencias en Educación Física, Deportes y Recreación, 36, 446-453.

Rodríguez, F. A., Valencia, S. C., Gaitán, E. M., González, S. A., \& León, J. D. (2017). Hábitos saludables, motivos y barreras en la realización de actividad física en estudiantes universitarios. Revista de Investigación: Cuerpo, Cultura y Movimiento, 7(1 y 2), 81-102. https://doi.org/10.15332/s22484418.2017.0001.05

Rosales, A., Fernández, M., Comunello, F., Mulargia, S., \& Ferran, N. (2017). «Older people and smartwatches, initial experiences». El profesional de la información, 3(26), 457-463. https://doi.org/ 10.3145/epi.2017.may.12.

Santos, G., Alberte, R., Colmenero, N., Vázquez, Y., De la Montaña, J., \& Míguez, M. (2017). Adherencia de la dieta de universitarios a la dieta mediterránea y su relación con la práctica de actividad física. En Romá, M.T.; Arroyo, H.V.; Aguiló, A. (coords.), Actas del Congreso Iberoamericano de Universidades Promotoras de la Salud (pp. 184-187). Alicante: Universidad Saludable, Universitat d'Alacant.

Sevil, J., Práxedes, A., Zaragoza, J., del Villar, F., \& García-Gónzalez, L. (2017). Barreras percibidas para la práctica de actividad física en estudiantes universitarios. Diferencias por género y niveles de actividad física. Universitas Psychologica, 16(4), 115. https://doi.org/10.11144/Javeriana.upsy16-4.bppa.

Talaván, J. (2015). ¿Tiene mi alumnado hábitos saludables? EmásF, Revista Digital de Educación Física, 36, 28-42.

Tauler, P., Riera, A., Bennasar-Veny, M., A, C., Borràs, P., \& Aguiló, A. (2017). Promoción de la actividad física en la Universidad de las Illes Balears mediante la instalación de microespacios deportivos. En Romá, M.T.; Arroyo, H.V.; Aguiló, A. (coords.), Actas del Congreso Iberoamericano de Universidades Promotoras de la Salud (pp. 168-172). Alicante: Universidad Saludable, Universitat d'Alacant.

Tong, H. L., Coiera, E., \& Laranjo, L. (2018). Using a Mobile Social Networking App to Promote Physical Activity: A Qualitative Study of Users' Perspectives. Journal of Medical Internet Research, 20(12). 1-13. doi: 10.2196/11439. 